\title{
Atividade física, sobrepeso e pressão arterial: associação independente e combinada em ad́olescentes
}

\author{
Physical activity, overweight and blood \\ pressure: independent and combined \\ association in adolescents
}

Afonso Larruscaim Cauduro'

Mauren Lúcia de Araújo Bergmann'

Gabriel Gustavo Bergmann¹

\section{RESUMO}

O objetivo do estudo foi analisar as associações independentes e combinadas entre a atividade física (AF), o sobrepeso e obesidade com a pressão arterial (PA) aumentada em adolescentes. Participaram do estudo 1.455 adolescentes (50,9\% do sexo feminino) de 10 a 17 anos de escolas públicas municipais e estaduais. O sobrepeso e a obesidade foram considerados através do índice de massa corporal (IMC) e a $\mathrm{AF}$ por questionário. Os adolescentes foram classificados como suficientemente ou insuficientemente ativos. O IMC e a AF tiveram suas categorias relacionadas para a realização da análise combinada. A PA foi aferida através do método auscultatório e classificada em normal ou aumentada. Os dados foram descritos e as associações testadas pelo Qui-quadrado e regressão logística binária. Identificou-se uma prevalência de sobrepeso, obesidade e AF insuficiente de 19,9\% (IC95\%: 17,8-22,0), 7,9\% (IC95\%: 6,5-9,3) e 68\% (IC95\%: 65,5-70,5), respectivamente. As prevalências de PA sistólica (PAS) e diastólica (PAD) aumentadas foram de 16,4\% (IC95\%: 14,4-18,4) e 18,5\% (IC95\%: 16,4-20,6), respectivamente. Na análise independente, apenas o sobrepeso e a obesidade permaneceram associados à PAS e PAD ( $<<005)$, tendo os adolescentes obesos mais chance de apresentarem PAS (OR: 9,96; IC95\%: 6,29-15,77) e PAD (OR: 5,68; IC95\%: 3,65-8,86) aumentadas. A análise combinada demonstrou que as prevalências de PA aumentada entre adolescentes com sobrepeso e obesos é reduzida entre os suficientemente ativos. Adolescentes com sobrepeso e obesos tem mais chance de terem PA aumentada. Esta chance é aumentada entre aqueles que são insuficientemente ativos.

\section{PALAVRAS-CHAVE}

Pressão arterial; Atividade motora; Índice de massa corporal; Jovens.

\begin{abstract}
The aim of this study was analyze the independent and combined associations between physical activity (PA), overweight and obesity to increased blood pressure (BP) in adolescents. They were part of the sample 1,455 adolescents (50.9\% female) aged 10 to 17 years old from public schools. Overweight and obesity were assessed by body mass index (BMI) and PA by questionnaire. Adolescents were classified as sufficiently or insufficiently actives. Categories of BMI and PA were cross-tabulated to the combined analysis be conducted. BP was assessed by auscultation and classified in normal or increased. Data were described and associations were tested by Chi-square test and binary logistic regression. Prevalence of overweight, obesity and insufficient PA of 19.9\% (CI95\%: 17.8-22.0), 7.9\% (CI95\%: 6.5-9.3) and 68.0\% (CI95\%: 65.5-70.5), respectively, were identified. Prevalence of increased systolic BP (SBP) and diastolic BP (DBP) were 16.4\% (CI95\%: 14.4-18.4) and $18.5 \%$ (CI95\%: 16.4-20.6) respectively. In the analyze independent, only overweight and obesity remained associated to increase SBP and DBP ( $\quad<0.05)$, having obese adolescents more likely to experience increased SBP (OR: 9.96; CI95\%: 6.29-15.77) and DBP (OR: 5.68; CI95\%: 3.65-8.86). The combined analyze showed that the prevalence of increased BP among overweight and obese adolescents is reduced in those are sufficiently actives. Overweight and obese adolescents are more likely to experience increased BP. These chance is enhanced between those are insufficiently active.
\end{abstract}

\section{KEYWORDS}

Blood Pressure; Motor Activity; Body Mass Index; Adolescent. 


\section{INTRODUCCÃO}

A hipertensão arterial é um problema de saúde com elevada prevalência na população ${ }^{1}$. Uma recente meta-análise envolvendo 55 estudos e mais de 120 mil adolescentes indicou que esta enfermidade também está presente neste grupo populacional, sugerindo que cerca de 10\% destes indivíduos apresenta PA aumentada ${ }^{2}$. No Brasil a prevalência encontrada em estudos com adolescentes é variável. Um estudo de revisão identificou valores de PA aumentada variando de $2,5 \%$ a $30,9 \%{ }^{3}$. Prevalência ainda mais alta $(44,7 \%)$ foi encontrada em um estudo realizado com indivíduos de seis a 18 anos $^{4}$.

As diferenças nas prevalências de PA aumentada encontradas entre os estudos podem ser explicadas, ao menos em parte, por questões metodológicas ${ }^{3}$. Contudo, independente das estratégias metodológicas utilizadas, as prevalências de PA aumentada encontradas entre adolescentes é preocupante. Este quadro é ainda mais grave em alguns subgrupos, como aqueles em situação de sobrepeso, obesos e fisicamente inativos ${ }^{4,5}$. O sobrepeso e a obesidade apresentam forte associação com a PA aumentada ${ }^{5,7-9}$. A atividade física (AF), por outro lado, não tem apresentado resultados conclusivos sobre sua associação com o desfecho nesta população ${ }^{6,7,9,10}$.

Quando estes fatores são analisados em conjunto, o sobrepeso e a obesidade permanecem associados à $\mathrm{PA}$ aumentada independente da $\mathrm{AF}^{6,11}$. $\mathrm{A} \mathrm{AF}$, contudo, apresenta resultados divergentes ${ }^{6,9,11}$. Esta heterogeneidade nos resultados referentes às associações entre $\mathrm{AF}$ e a $\mathrm{PA}$ aumentada em adolescentes, independentes do sobrepeso e da obesidade, indica que uma lacuna ainda persiste em relação a esta temática. Análises envolvendo estes fatores de forma combinada poderiam disponibilizar informações adicionais para a melhor compreensão das associações com a PA aumentada. Porém, estudos com este propósito não foram encontrados na literatura. Diante disto, o objetivo deste estudo foi analisar as associações independentes e combinadas entre a AF, o sobrepeso e obesidade com a PA aumentada em adolescentes.

\section{MÉTODOS}

\section{População e amostra}

Este estudo transversal descritivo foi desenvolvido utilizando informações do banco de dados do projeto "Atividade física e fatores associados em escolares de 10 a 17 anos de Uruguaiana, RS". A amostra foi selecionada aleatoriamente em múltiplas fases. O cálculo para o tamanho mínimo da amostra foi realizado considerando os seguintes critérios: o número de alunos matriculados regularmente no ano de 2011 na faixa etária do estudo $(n=15.210)$; prevalência de $50 \%$, considerando um estudo com múltiplos desfechos; intervalo de confiança de $95 \%$; erro aceitável de 3 pontos percentuais; e, percentual de perdas e recusas de $20 \%$. Com estes critérios foi estimada a necessidade de selecionar 1.196 escolares.

Para a seleção dos escolares, inicialmente o município foi divido em cinco áreas. A zona urbana foi dividida em quatro regiões (quadrantes) e zona rural foi considerada como um todo. Para alcançar o número de escolares estimados pelo cálculo amostral mantendo a proporcionalidade por áreas urbanas e zona rural, foi necessário selecionar nove escolas da zona urbana (duas em três 
áreas e três na área restante) e uma escola da zona rural. A seleção das escolas ocorreu por sorteio simples. Todos os alunos das turmas de quinta série do ensino fundamental ao terceiro ano do ensino médio das dez escolas foram convidados a participar do estudo e receberam informações sobre sua realização. Todos adolescentes que no dia agendado para a coleta de dados apresentaram o termo de consentimento livre e esclarecido e manifestaram interesse participaram do estudo. Dentre aqueles que foram incluídos no estudo e tiveram suas informações coletadas, foram excluídos das análises os que estavam fora da faixa etária de interesse.

\section{Aspectos éticos}

O projeto de pesquisa da qual as informações foram utilizadas para a realização deste estudo foi analisado e aprovado pelo comitê de ética em pesquisa da Universidade Federal do Pampa (protocolo 042/2010). Todos os adolescentes que participaram do estudo apresentaram o termo de consentimento livre e esclarecido assinado por um responsável e assinaram o termo de assentimento do menor.

\section{Variáveis e procedimentos de coleta de dados}

Para a realização do presente estudo foram utilizados dados de índice de massa corporal, de AF e PA. O IMC foi calculado com os valores de massa corporal $(\mathrm{kg})$ e estatura $(\mathrm{m})$ (massa corporal/estatura ${ }^{2}$ ) e os resultados foram categorizados em peso normal, sobrepeso ou obesidade conforme pontos de corte específicos por sexo e idade ${ }^{12}$. A AF foi estimada por meio do questionário para AF para crianças e a adolescentes (Physical Activity Questionnaire for Older Children $P A Q-C^{13}$ and Adolescents $\left.P A Q-A^{14}\right)$ adaptado para a realidade brasilei$\mathrm{ra}^{15}$. Os resultados são expressos em um escore contínuo que, dividido pelo número de questões, permite a criação de cinco categorias de AF conforme a proposta original ${ }^{13,14}$. Indivíduos classificados dentro das duas primeiras categorias foram considerados insuficientemente ativos. Os classificados dentro das outras três categorias foram considerados suficientemente ativos.

A PA foi considerada a partir dos valores de pressão arterial sistólica (PAS) e pressão arterial diastólica (PAD). As medidas foram realizadas por avaliadores previamente treinados e capacitados para a coleta utilizando método auscultatório. Todos os instrumentos utilizados (estetoscópios, manguitos e esfigmomanômetros) foram aferidos pelo Instituto Nacional de Metrologia. Os manguitos e os esfigmomanômetros eram de dimensões adequadas ao tamanho do braço dos adolescentes. Os adolescentes não realizaram AF nos 30 minutos anteriores à coleta e estavam em repouso a pelo menos 10 minutos antes da aferição. As medidas foram realizadas em triplicata (cerca de dois minutos de intervalo entre as medidas) no braço direito com os adolescentes sentados e relaxados ${ }^{3}$. A PAS foi determinada no aparecimento do primeiro som de Korotkof e a PAD no desaparecimento do mesmo. Para a categorização da PA, levou-se em consideração o sexo a idade e o percentil da estatura de acordo com The Fourth Report on Diagnosis, Evaluation, and Treatment of High Blood Pressure in Children and Adolescents ${ }^{16}$ e os adolescentes foram classificados em normalidade, pré-hipertensão e hipertensão. Para a realização das análises as categorias pré-hipertenso e hipertenso foram agrupadas e consideradas como PAS e PAD aumentadas.

Para a realização das análises combinadas foi criada uma nova variável cruzando as categorias do IMC e da AF (IMC/AF) ("peso normal/suficientemen- 
te ativo", "peso normal/insuficientemente ativo", "sobrepeso/suficientemente ativo", "sobrepeso/insuficientemente ativo", "obeso/suficientemente ativo", "obeso/insuficientemente ativo"). Além das descritas anteriormente, também foram utilizadas as variáveis nível socioeconômico, estimada com o instrumento de Classificação Econômica Brasil ${ }^{20}$ e sua classificação em cinco níveis de "A" a "E"; sexo (masculino ou feminino); idade (anos completos); e, zona de moradia (rural ou urbana). Estas variáveis foram utilizadas na análise multivariável como variáveis de ajuste.

\section{Análise dos dados}

Inicialmente os dados foram descritos com a utilização das frequências absolutas e relativas (proporções) em cada uma das variáveis analisadas seguidas pelo cálculo do intervalo de confiança de 95\% (IC95\%). A frequência de adolescentes com PAS e PAD aumentadas nas categorias do IMC e da AF foi analisada utilizando-se os testes Qui-quadrado para tendência linear e para heterogeneidade. Para a análise independente, foi utilizada a regressão logística binária tendo a PAS e PAD dicotomizadas como desfecho, o IMC e a AF como variáveis independentes, e o nível socioeconômico, o sexo, a idade e a zona de moradia como variáveis de ajuste. Para as análises combinadas, a frequência de adolescentes com PAS e PAS aumentadas nas seis categorias do IMC/AF foi analisada utilizando-se os testes Qui-quadrado para tendência linear. A associação ajustada entre a IMC/AF e as PAS e PAD aumentadas foi realizada com a utilização da regressão logística binária. Todas as análises foram realizadas no programa SPSS para Windows, versão 20.0 levando em consideração um IC95\% e p $\leq 0,05$.

\section{RESULTADOS}

Um total de 1.455 escolares, 50,9\% (741) meninas, participou do estudo. As prevalências de sobrepeso, obesidade, AF insuficiente e PAS e PAD aumentadas foram de 19,9\% (IC95\%: 17,8-22,0), 7,9\% (IC95\%: 6,5-9,3), 68,0\% (IC95\%: 65,5-70,5), 16,4\% (IC95\%: 14,4-18,4) e 18,5\% (IC95\%: 16,4-20,6), respectivamente. $\mathrm{Na}$ tabela 1 são apresentadas as características sociodemográficas da amostra analisada.

Nas análises do Qui-quadrado os resultados apontaram uma frequência significativamente superior de adolescentes classificados com PAS e PAD aumentada entre aqueles com sobrepeso e obesidade e com PAD aumentada entre os insuficientemente ativos (tabela 2). Os resultados da análise de regressão logística indicaram que adolescentes com sobrepeso e obesos têm mais chance $(\mathrm{p}<0,05)$ de apresentarem PAS e PAD aumentadas que aqueles com peso normal independente do nível de $\mathrm{AF}$ (tabela 3). A AF não se associação à $\mathrm{PA}$ aumentada na análise ajustada.

Com relação à associação combinada entre o IMC e AF com a PA aumentada, os resultados indicaram que a frequência de adolescentes com PAS e PAD aumentadas é crescente entre as categorias do IMC sendo mediada pela AF (figura 1). Adolescentes obesos e insuficientemente ativos têm em torno de 15 e sete vezes mais chance de apresentarem PAS e PAD aumentadas, respectivamente, que seus pares com peso normal e suficientemente ativos (tabela 4). 
TABELA 1 - Características sociodemográficas de adolescentes de Uruguaiana/RS, 2011.

\begin{tabular}{|c|c|c|c|}
\hline Variáveis & $n$ & $\%$ & (IC95\%) \\
\hline \multicolumn{4}{|l|}{ Sexo $(n=1455)$} \\
\hline Masculino & 714 & 49,1 & $(46,5-51,7)$ \\
\hline Feminino & 741 & 50,9 & $(48,3-53,5)$ \\
\hline \multicolumn{4}{|l|}{ Idade $(n=1455)$} \\
\hline 10 anos & 79 & 5,4 & $(4,2-6,6)$ \\
\hline 11 anos & 209 & 14,4 & $(12,6-16,2)$ \\
\hline 12 anos & 205 & 14,1 & $(12,3-15,9)$ \\
\hline 13 anos & 232 & 15,9 & $(14,0-17,8)$ \\
\hline 14 anos & 211 & 14,5 & $(12,7-16,3)$ \\
\hline 15 anos & 221 & 15,2 & $(13,3-17,0)$ \\
\hline 16 anos & 174 & 12,0 & $(10,3-13,7)$ \\
\hline 17 anos & 124 & 8,5 & $(7,1-9,9)$ \\
\hline \multicolumn{4}{|c|}{ Zona de moradia ( $\mathrm{n}=1427$ ) } \\
\hline Urbana & 1296 & 90,8 & $(89,2-92,4)$ \\
\hline Rural & 131 & 9,2 & $(8,7-9,7)$ \\
\hline \multicolumn{4}{|c|}{ Nível socioeconômico (n=1278) } \\
\hline Classe B & 263 & 20,6 & $(18,4-22,8)$ \\
\hline Classe C & 817 & 63,9 & $(61,3-66,5)$ \\
\hline Classe $\mathrm{D} / \mathrm{E}^{*}$ & 198 & 15,5 & $(13,5-17,5)$ \\
\hline
\end{tabular}

n: tamanho da amostra; \%: proporção da amostra; IC95\%: intervalo de confiança de $95 \%$. ${ }^{*} \mathrm{Ne}$ nhum escolar foi classificado no nível socioeconômico $\mathrm{A}$. Devido ao reduzido número de escolares classificados como pertencentes ao nível socioeconômico E, esta categoria foi agrupada à categoria nível socioeconômico D. Nenhum escolar foi classificado no nível socioeconômico A.

TABELA 2 - Prevalência de adolescentes com pressão arterial sistólica e diastólica aumentadas de acordo com índice de massa corporal e a atividade física. Uruguaiana/RS, 2011.

\begin{tabular}{lrccccc}
\hline & \multicolumn{2}{c}{ PAS aumentada } & \multicolumn{3}{c}{ PAD aumentada } \\
\cline { 2 - 7 } & $\mathrm{n}$ & $\%($ IC95\%) & $\mathrm{p}$ & $\mathrm{n}$ & $\%($ IC95\%) & $\mathrm{p}$ \\
\hline Índice de massa corporal ${ }^{*}(\mathrm{n}=1339)$ & & $<0,001$ & & & $<0,001$ \\
\hline Peso normal & 964 & $9,2(7,4-11,0)$ & & 963 & $13,2(11,1-15,3)$ & \\
\hline Sobrepeso & 268 & $29,5(24,0-35,0)$ & & 262 & $27,9(22,5-33,3)$ & \\
\hline Obesidade & 107 & $48,6(39,1-58,1)$ & & 107 & $43,0(33,6-52,4)$ & \\
\hline Atividade Física** $(\mathrm{n}=1251)$ & & & 0,066 & & & 0,044 \\
\hline Suficientemente ativos & 405 & $13,8(10,4-17,6)$ & & 403 & $15,9(12,3-19,5)$ & \\
\hline Insuficientemente ativos & 846 & $18,0(15,4-20,6)$ & & 841 & $20,7(18,0-23,4)$ & \\
\hline
\end{tabular}

PAS: pressão arterial sistólica; PAD: pressão arterial diastólica; $\mathrm{n}$ : tamanho absoluto da amostra; $\%$ : tamanho relativo da amostra; IC95\%: intervalo de confiança de $95 \%$; *Qui-quadrado para tendência; ${ }^{* *}$ Qui-quadrado para heterogeneidade.

TABELA 3 - Associação ajustada entre pressão arterial sistólica e diastólica, estado nutricional e nível de atividade física em adolescentes de 10 a 17 anos. Uruguaiana/RS, 2011.

\begin{tabular}{lcccccc}
\hline & \multicolumn{3}{c}{ PAS aumentada } & \multicolumn{3}{c}{ PAD aumentada } \\
\hline & OR & IC95\% & $\mathrm{p}$ & OR & IC95\% & $\mathrm{p}$ \\
\hline Índice de massa corporal* $(\mathrm{n}=1127)$ & & & & & \\
\hline Peso normal & 1 & - & - & 1 & - & - \\
\hline Sobrepeso & 4,29 & $2,94-6,25$ & $<0,001$ & 2,83 & $1,97-4,06$ & $<0,001$ \\
\hline Obeso & 9,39 & $5,71-15,44$ & $<0,001$ & 5,57 & $3,45-8,98$ & $<0,001$ \\
\hline Atividade física** $(\mathrm{n}=1107)$ & & & & & & \\
\hline Suficientemente ativo & 1 & - & - & 1 & - & - \\
\hline Insuficientemente ativo & 1,24 & $0,84-1,84$ & 0,272 & 1,37 & $0,95-1,99$ & 0,093 \\
\hline
\end{tabular}

$\mathrm{n}$ : tamanho absoluto da amostra; PAS: pressão arterial sistólica; PAD: pressão arterial diastólica; OR: odds ratio; p: significância; *Ajustada para atividade física, sexo, idade, zona de moradia e nível socioeconômico;

** Ajustada para o índice de massa corporal, sexo, idade, zona de moradia e nível socioeconômico. 


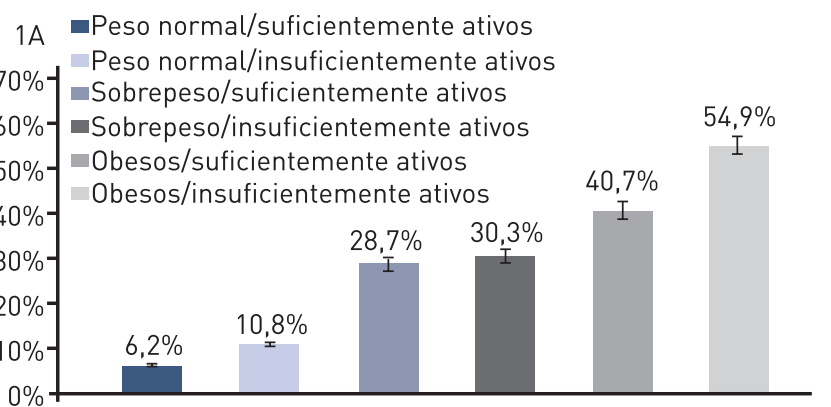

PAS Aumentada
$1 \mathrm{~B}$

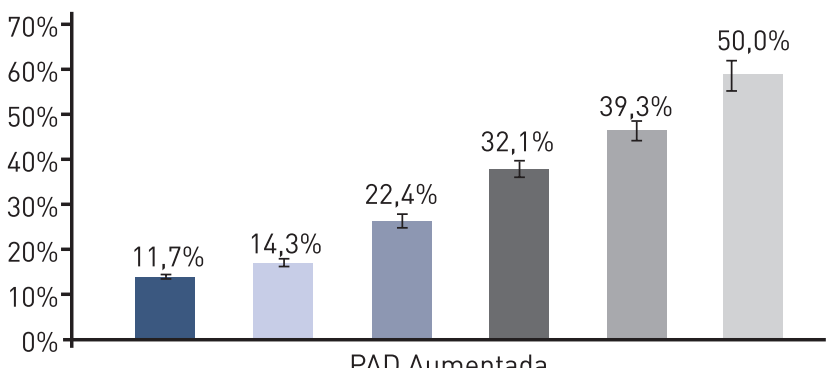

PAD Aumentada

FIGURA 1 - Frequência de adolescentes com pressão arterial sistólica (figura 1a) e diastólica (figura 1b) aumentadas de acordo com as categorias cruzadas do estado nutricional e do nível de atividade física. Uruguaiana/RS, 2011.

TABELA 4 - Associação ajustada entre pressão arterial sistólica e diastólica e as categorias cruzadas do índice de massa corporal e da atividade física em adolescentes de 10 a 17 anos. Uruguaiana/RS, 2011.

\begin{tabular}{lcccccc}
\hline & \multicolumn{3}{c}{ PAS elevada } & & \multicolumn{3}{c}{ PAD elevada } \\
\hline Estado nutricional/Atividade física & OR & IC95\% & P & OR & IC95\% & P \\
Peso normal/suficientemente ativos & 1 & - & - & 1 & - & - \\
\hline Peso normal/insuficientemente ativos & 1,56 & $0,88-2,77$ & 0,125 & 1,23 & $0,76-1,98$ & 0,394 \\
Sobrepeso/suficientemente ativos & 5,51 & $2,96-10,26$ & $<0,001$ & 2,31 & $1,18-4,52$ & 0,014 \\
Sobrepeso/insuficientemente ativos & 6,61 & $3,34-13,07$ & $<0,001$ & 3,78 & $2,20-6,52$ & $<0,001$ \\
Obesos/suficientemente ativos & 9,17 & $3,55-23,68$ & $<0,001$ & 4,90 & $2,0211,85$ & $<0,001$ \\
\hline Obesos/insuficientemente ativos & 14,99 & $7,37-30,49$ & $<0,001$ & 7,21 & $3,79-13,72$ & $<0,001$ \\
\hline
\end{tabular}

PAS: pressão arterial sistólica; PAD: pressão arterial diastólica; OR: odds ratio; p: significância; Análises ajustadas para sexo, idade, zona de moradia e nível socioeconômico.

\section{DISCUSSÃO}

O principal achado do presente foi identificar que a AF atenua a prevalência de PA aumentada entre os adolescentes em situação de sobrepeso e obesidade. A prevalência de adolescentes com PAS aumentada entre os obesos suficientemente ativos foi quase 15 pontos percentuais menor que aqueles com a mesma classificação do IMC, mas insuficientemente ativos. Em relação à PAD aumentada, a redução foi de cerca de 10 pontos percentuais.

Os resultados das análises descritivas indicaram um quadro preocupante, mas não surpreendente, de elevadas prevalências de adolescentes com PA aumentada, insuficientemente ativos, e com sobrepeso e obesidade. Em estudo realizado com adolescentes de 17 a 19 anos, Corrêa-Neto et al. ${ }^{17}$ encontraram prevalência de PA aumentada de $19,4 \%$. No estudo de Constanzi et al. ${ }^{5}$ com escolares de 7 a 12 anos de idade a prevalência foi de 13,8\%. Com relação à AF, estudos que utilizaram o mesmo instrumento e procedimento de classificação encontraram prevalências ainda superiores de adolescentes insuficientemente $\operatorname{ativos}^{15,18}$. No que se refere ao IMC, estudos realizados em cidades das regiões Nordeste $^{19}$, Sudeste ${ }^{20}$ e Sul ${ }^{21}$ do país encontraram prevalências de sobrepeso e obesidade similares às do presente estudo. Além dos prejuízos observáveis já nos anos da adolescência decorrentes de níveis insuficientes de AF e do sobrepeso e obesidade ${ }^{22-25}$, a AF, o IMC e a PA apresentam certa estabilidade deste período para a vida adulta ${ }^{26-28}$, indicando que adolescentes com $\mathrm{AF}$ insuficiente, em situação de sobrepeso e obesidade, e com PA aumentada têm mais chance de permanecerem desta forma quando se tornarem adultos. Estes resultados reforçam a necessidade de criação de estratégias para o aumento no nível de $\mathrm{AF}$ e redução da prevalência de sobrepeso e obesidade em adolescentes. 
Nas análises de associação independentes entre o IMC, AF e PA os resultados deste estudo revelaram uma prevalência significativamente maior de adolescentes com PA aumentada entre aqueles em situação de sobrepeso e obesidade, independente da $\mathrm{AF}$ e de características demográficas. Uma série de outros estudos também encontrou associação direta entre o IMC e a PA aumentada independente do nível de $\mathrm{AF}$ dos adolescentes ${ }^{6,11,17}$. As razões para adolescentes com sobrepeso e obesos terem mais chance de apresentarem níveis pressóricos mais altos que seus pares com peso normal não estão totalmente esclarecidas e envolvem uma série de possíveis mecanismos. Dentre os principais estão o aumento da resistência vascular sistêmica, o aumento da atividade do sistema nervoso simpático, a resistência à insulina e disfunção endotelial ${ }^{29}$.

Embora na análise de associação sem ajuste a prevalência de adolescentes com PA aumentada tenha sido maior entre os insuficientemente ativos (não significativo para a PAS), diferentemente do IMC, na análise ajustada a $\mathrm{AF}$ não apresentou associação significativa com a PA. Estes resultados vão ao encontro de outros estudos que também não identificaram maiores frequências de adolescentes com PA aumentada entre aqueles classificados como insuficientemente ativos ${ }^{6,11,17}$.

Mesmo sem se associar com a PA, a AF não deve ser interpretada como um comportamento sem efeito neste desfecho em adolescentes. Os resultados das análises combinadas indicaram que a frequência de PA aumentada é menor entre os adolescentes com sobrepeso e obesos suficientemente ativos em relação aos insuficientemente ativos. Este resultado pode ser considerado como o principal achado deste estudo, indicando que a prática de AF pode amenizar os prejuízos do sobrepeso e da obesidade relacionados à PA de adolescentes.

Estes achados corroboram os resultados apresentados por Torrance et al. ${ }^{29}$, que sugerem que a prática de $\mathrm{AF}$ pode exercer um papel mediador na associação entre sobrepeso, obesidade e PA elevada em adolescentes. Apesar das evidências não serem conclusivas, a prática de $\mathrm{AF}$ parece atuar de forma positiva na função endotelial, contribuindo para o controle da $\mathrm{PA}^{29}$. Neste sentido, um estudo de intervenção indicou que prática regular de AF aeróbicas é capaz de reduzir os valores de PAS, PAD e a prevalência de PA elevada, além de reduzir os valores de IMC, gordura abdominal, aumentar o consumo máximo de oxigênio, e reduzir a espessura da íntima média da artéria carótida e a rigidez arterial em adolescentes obesos ${ }^{30}$. Estas evidências reforçam a necessidade de elaboração de ações de incentivo e de programas disponibilizando opções para que adolescentes pratiquem $\mathrm{AF}$ regularmente.

Embora resultados importantes tenham sido disponibilizados, algumas limitações devem ser consideradas ao interpretá-los. A prevalência de PA aumentada encontrada deve ser considerada a partir da opção de agrupamento das categorias pré-hipertensão e hipertensão. Caso o desfecho tivesse sido considerado apenas pela categoria hipertensão as prevalências seriam diferentes e talvez esta modificação interferisse nos resultados das análises. Por ser um estudo transversal, não é possível atribuir relação de causa e efeito aos resultados das associações realizadas. A utilização de questionário para a medida da AF é outra limitação do estudo. Por exemplo, cientes dos benefícios da AF para a saúde, adolescentes sabedores de sua condição de PA aumentada podem relatar ter praticado uma quantidade de $\mathrm{AF}$ maior do que aquela realmente realizada na semana anterior à coleta dos dados. Apesar de ter sido 
realizada seguindo todas as recomendações, a medida da PA em um único dia é uma limitação, pois pode não identificar os reais valores ${ }^{3}$. Por fim, fatores potencialmente associados ao IMC e à PA em adolescentes, como estágio maturacional ${ }^{20}$, genética ${ }^{2,16} \mathrm{e}$ hábitos alimentares ${ }^{2,16}$ não foram analisados.

Mesmo com as limitações discutidas acima, é importante destacar que os achados deste estudo são fortalecidos pelos procedimentos utilizados para a seleção da amostra. O tamanho confere precisão e a forma de seleção representatividade. Estas características aumentam o poder das análises e a validade interna do estudo.

Em conclusão, a prevalência de adolescentes com PA aumentada é elevada na amostra analisada, sendo maior entre aqueles em situação de sobrepeso e os obesos. A AF diminui a chance de adolescentes com sobrepeso e obesos de apresentarem PA aumenta, sugerindo que mesmo este grupo pode se beneficiar com a prática de AF.

\section{Contribuição dos autores}

ALC participou da análise e interpretação dos dados, da redação do artigo e aprovou a versão final ser publicada. MLAB e GGB participaram de todas as etapas do estudo e aprovaram sua versão final.

\section{REFERÊNCIAS}

1. Sociedade Brasileira de Cardiologia/Sociedade Brasileira de Hipertensão/Sociedade Brasileira de Nefrologia. VI Diretrizes Brasileiras de Hipertensão. Arq Bras Cardiol. 2010;95(1 supl.1):1-51.

2. de Moraes ACF, Lacerda MB, Moreno LA, Horta BL, Carvalho HB. Prevalence of High Blood Pressure in 122,053 Adolescents: A Systematic Review and Meta-Regression. Medicine. 2014;93(27):1-10.

3. Magalhães MGPA, Oliveira LMFT, Christofaro DGD, Ritti-Dias RM. Prevalência de pressão arterial elevada em adolescentes brasileiros e qualidade dos procedimentos metodológicos empregados: revisão sistemática. Rev bras epidemiol 2013;16(4):849-859.

4. Araújo TL, Lopes MVO, Cavalcante TF, Guedes NG, Moreira RP, Chaves ES, et al. Análise de indicadores de risco para hipertensão arterial em crianças e adolescentes. Rev Esc Enferm. 2008; 42:120-6.

5. Costanzi CB, Halpern R, Rech RR, Bergmann ML, Alli LR, de Mattos AP. Associated factors in high blood pressure among schoolchildren in a middle size city, southern Brazil. J Pediatr. 2009;85(4):335-40.

6. Christofaro DGD, Ritti-Dias RM, Chiolero A, Fernandes RA, Casonatto J, de Oliveira AR. Physical activity is inversely associated with high blood pressure independently of overweight in Brazilian adolescents. Scand J Med Sci Sports. 2013;23(3):317-22.

7. Marty K, Wolff C, Morgan I. Overweight, Diet, Physical Activity, and Hypertension in Low-Income School-Aged Children. Calif J Health Promot. 2006;4(2):47-58.

8. de Souza MGB, Rivera IR, da Silva MAM, Carvalho ACC. Relação da Obesidade com a Pressão Arterial Elevada em Crianças e Adolescentes. Arq Bras Cardiol. 2010;94(6):714-9.

9. Hayes HM, Eisenmann JC, Pfeiffer K, Carlson JJ. Weight status, physical activity, and vascular health in 9- to 12-year-old children. J Phys Act Health. 2013;10(2):205-10.

10. Durrani AM, Fatima W. Effect of Physical Activity on Blood Pressure Distribution among School Children. Adv in Public Health. 2015;2015:1-4.

11. Ortiz RM, Rodriguez R, Depaoli S, Weffer SE. Increased Physical Activity Reduces the Odds of Elevated Systolic Blood Pressure Independent of Body Mass or Ethnicity in Rural Adolescents. J Hypertens. 2014;3(3):1-8.

12. Cole TJ, Bellizzi MC, Flegal KM, Dietz WH. Establishing a standard definition for child overweight and obesity worldwide: international survey. BMJ. 2000;320(7244):1240-3. 
13. Crocker PRE, Bailey DA, Faulkner RA, Kowalski KC, Mcgrath R. Measuring general levels of physical activity: Preliminary evidence for the Physical Activity Questionnaire for Older Children. Med Sci Sports Exerc. 1997;29(10):1344-9.

14. Kowalski KC, Crocker PRE, Faulkner RA. Validation of the physical activity questionnaire for older children. Pediatr Exerc Sci. 1997;9(2):174-86.

15. Silva RC, Malina RM. Level of physical activity of in adolescents of Niterói, Rio de Janeiro, Brazil. Cad Saúde Pública. 2000;16(4):1091-7.

16. NHBPEP. National High Blood Pressure Education Program Working Group on High Blood Pressure in Children and Adolescent. The Fourth report on the diagnosis, evaluation, end treatment of high blood pressure in children and adolescents. Pediatrics. 2004;114(2):555-76.

17. Corrêa Neto VG, Sperandei S, Silva LAI, Maranhão Neto GA, Palma A. Hipertensão arterial em adolescentes do Rio de Janeiro: prevalência e associação com atividade física e obesidade. Cien Saude Colet. 2014;19(6):1699-708.

18. Rivera IR, da Silva MAM, Silva RDTA, de Oliveira BAV, Carvalho ACC. Atividade Física, Horas de Assistência à TV e Composição Corporal em Crianças e Adolescentes. Arq Bras Cardiol. 2010;95(2):159-65.

19. Ramires EKNM, de Menezes RCE, Oliveira JS, Oliveira MAA, Temoteo TL, LongoSilva G, et al. Estado nutricional de crianças e adolescentes de um município do semiárido do Nordeste brasileiro. Rev Paul Pediatr. 2014;32(3):200-7.

20. Castilho SD, Nucci LB. Age at menarche in schoolgirls with and without excess weight. J Pediatr (Rio J). 2015;91(1):75-80.

21. Rech RR, Halpern R, Constanzi CB, Bergmann MLA, Alli LR, de Mattos AP, et al. Prevalência de obesidade em escolares de 7 a 12 anos de uma cidade Serrana do RS, Brasil. Rev Bras Cineantropom Desempenho Hum. 2010;12(2):90-7.

22. Guedes DP, Guedes JERP, Barbosa DS, de Oliveira JA, Stanganelli LCR. Fatores de Risco Cardiovasculares em Adolescentes: Indicadores Biológicos e Comportamentais. Arq Bras Cardiol. 2006;86(6):439-50.

23. Silva D, Antunes H. Psychosocial correlates of overweight and obesity in infancy. Journal of Human Growth and Development. 2012;22(2):179-86.

24. Romero A, de Medeiros MJ, Borges CA, Romero SCS, Slater B. Associação entre atividade física e marcadores bioquímicos de risco para doença cardiovascular em adolescentes de escolas públicas de Piracicaba. Rev Bras Ativ Fis Saúde. 2013;18(5):614-22.

25. López MS1, de Jensen ANM, Claudia Nora Mir CN, de Pianesi MEI, Manulak MA. Abdominal adiposity, insulin resistance, hypertension and urinary sodium in obese adolescents. Acta Bioquím Clín Latinoam. 2014;48(3):301-10.

26. Bao W, Threefoot SA, Srinivasan SR, Berenson GS. Essential hypertension predicted by tracking of elevated blood pressure from childhood to adulthood: the Bogalusa Heart Study. Am J Hypertens. 1995;8(7):657-65.

27. Singh AS, Mulder C, Twisk JW, van Mechelen W, Chinapaw MJ. Tracking of childhood overweight into adulthood: a systematic review of the literature. Obes Rev. 2008;9(5):474-88.

28. Telama R, Yang X, Leskinen E, Kankaanpää A, Hirvensalo M, Tammelin T, et al. Tracking of physical activity from early childhood through youth into adulthood. Med Sci Sports Exerc. 2014;46(5):955-62.

29. Torrance B, McGuire KA, Lewanczuk R, McGavock J. Overweight, physical activity and high blood pressure in children: a review of the literature. Vasc Health Risk Manag. 2007:3(1) 139-49.

30. Farpour-Lambert NJ, Aggoun Y, Marchand LM, Martin XE, Herrmann FR, Beghetti M. Physical activity reduces systemic blood pressure and improves early markers of atherosclerosis in pre-pubertal obese children. J Am Coll Cardiol. 2009;54(25):2396-406.

\section{ENDERECO PARA}

CORRESPONDÊNCIA

GABRIEL GUSTAVO BERGMANN

BR 472 - Km 592 - Caixa Postal 118

- Uruguaiana - RS - CEP: 97500-970

Universidade Federal do Pampa -

RECEBIDO 14/07/2015

Campus Uruguaiana. Tel: (55) 91993926

E-mail: gabrielbergmanndunipampa.edu.br 